\title{
System Analysis of the Expected Electrodynamic Tether Performance for the ProSEDS Mission
}

\author{
Keith R Fuhrhop and Brian E. Gilchrist, University of Michigan \\ Sven G Bilén, The Pennsylvania State University \\ Nestor R. Voronka, Tethers Unlimited Inc.
}

\begin{abstract}
This paper reviews some expected systems performance aspects of NASA's ProSEDS (Propulsive Small Expendable Deployer System) electrodynamic (ED) tether mission after recently being required to lower its initial orbit from $360 \mathrm{~km}$ to $285 \mathrm{~km}$. In addition, the ProSEDS tether, which has conductive and non-conductive sections, shortened its nonconductive section thereby reducing overall tether length from 15-km to 12-km long. The International Reference Ionosphere (IRI) model is not as accurate as previously predicted when the altitude is less than $300 \mathbf{~ k m}$ and it was found that a factor of 0.65 should be multiplied to the electron plasma density on the IRI 1990 model to compensate for this effect. The ED characteristics of ProSEDS are being theoretically predicted using software called TEMPEST developed at the University of Michigan. Using OML (Orbital Motion Limited) theory for tether collection TEMPEST demonstrated that ProSEDS will de-orbit in 90 hours instead of the originally predicted 160 hours. The induced EMF (electromotive force) range will remain approximately the same from $400 \mathrm{~V}$ to $1000 \mathrm{~V}$ as will the collected current range, which varies according to altitude. Also, it takes $\mathbf{5 0}$ hours for the atmospheric drag to become stronger than the electromagnetic drag from the tether at $285 \mathrm{~km}$ as opposed to the 130 hours at $360 \mathrm{~km}$. Various errors in the potential measurement of ProSEDS stem from: (1) the electron sheath at the upper end of the tether, (2) a "phantom current" occurring throughout the tether, and (3) the measurement of the potential used for current collection on the nozzle of the Delta II module. The "phantom current" also causes an extra $2 \mathrm{~km}$ per day de-orbit. In addition, there is a $0.733-\mathrm{mA}$ theoretical current that travels into the Delta II from the tether that must be emitted. This potential is plotted versus the atmospheric density to show how much current is collected. The total potential error in the ProSEDS system ranges from 4-71\% without hollow cathode operating, depending on the density of the electron plasma.
\end{abstract}

Introduction

NaSA's Propulsive Small Expendable Deployer System (ProSEDS) space experiment will demonstrate the use of an electrodynamic-tether propulsion system to generate thrust in space by decreasing the orbital altitude of a Delta II expendable launch vehicle second stage. Electrodynamic-tether thrusters work by virtue of the force that the Earth's magnetic field exerts on a wire carrying an electrical current-achieving thrust without the expenditure of propellant. ProSEDS, which is now to launch on an Air Force GPS Satellite replacement mission later in 2003 or in 2004, will use the flight-proven Small Expendable Deployer System (SEDS) to deploy a tether (5-km bare wire plus now 7-km non-conducting Dyneema) from a Delta II second stage to achieve $\sim 0.4 \mathrm{~N}$ drag thrust.

ProSEDS will utilize the tether-generated current to provide limited spacecraft power. The ProSEDS instrumentation includes Langmuir probes and Differential Ion Flux Probes, which will determine the characteristics of the ambient ionospheric plasma. Two Global Positioning System (GPS) receivers will be used (one on the Delta and one on its University of Michigan student-built endmass) to help determine tether dynamics and to limit transmitter operations to occasions when the spacecraft is over selected ground stations. The flight experiment is a precursor to a more ambitious electrodynamic-tether upper-stage demonstration mission, which will be capable of orbit raising, lowering, and inclination changes - all using electrodynamic thrust. An immediate application of ProSEDS technology is for the removal of spent satellites for orbital debris mitigation. In addition to the use of this technology to provide orbit transfer and debris mitigation it may also be an attractive option for future missions to Jupiter and any other planetary body with a magnetosphere.

In spring 2003, after the Columbia Space Shuttle accident, it was determined that the ProSEDS mission should change its starting altitude and alter the length of the tether to eliminate even the remotest chance of a tether section approaching the International Space Station. These required modifications delayed the ProSEDS launch from its planned date of late March 2003. They also resulted in the change in expected performance for some aspects of the mission. However, the primary mission objectives are still expected to be achieved. ${ }^{1}$ 
In this paper, we will discuss the expected changes in electrodynamic-tether (ED) performance due to changes in initial conditions. This will address basic performance parameters such as generated electromotive force (EMF), tether maximum current, and expected altitude change due to the ED tether forces and atmospheric drag effects. Next, we will also discuss previously unpublished results describing the challenges of tether voltage measurements with a bare tether. ${ }^{2}$ We will also add the assessment of ion current collection to the engine nozzles, which is important when the tether is in its "open-circuit" condition. Various aspects of the nozzle collection are discussed, including the direction of plasma flow, the plasma sheath size, and various environmental conditions. During the open-circuit mode there is still a very small negative current that gets through the large resistor. These electrons must be emitted from the nozzle in order to maintain charge neutrality. The nozzle must supply the voltage to maintain this charge neutralization.

\section{ProSEDS}

The EMF generated along the ProSEDS tether for its west-to-east orbit causes electrons to move down the length of the tether. This EMF is created by the velocity of the tether moving across the Earth's magnetic field and any co-rotating plasma and is governed by the equation

$$
V_{\text {emf }}=\left(v \times B_{\text {North }}\right) \cdot d l .
$$

This EMF dictates the flow of electrons through the length of the tether across the Earth's magnetic field creates another force that produces a drag thrust that helps de-orbit the system as given by

$$
d F=d l \cdot I \times B_{\text {North }} .
$$

On a bare tether, as in the case of ProSEDS, the current collected varies along the length of the tether because all of the bare tether length acts as part of collection surface. The changing current, combined with the efficiencies of the electron collection and ejection, control the drag force on the system. Calculated values are displayed in the following section.

In order for ProSEDS to be considered successful, a number of declared objectives must be accomplished. The primary objectives are to 1) demonstrate significant electrodynamic-tether thrust in space and 2) measure the current collection performance of the bare electrodynamic tether under varied ionospheric conditions Mission Changes

The direct results of the required modifications are that the lifetime of the mission will be reduced. ProSEDS will start off at a lower altitude where the atmospheric drag is

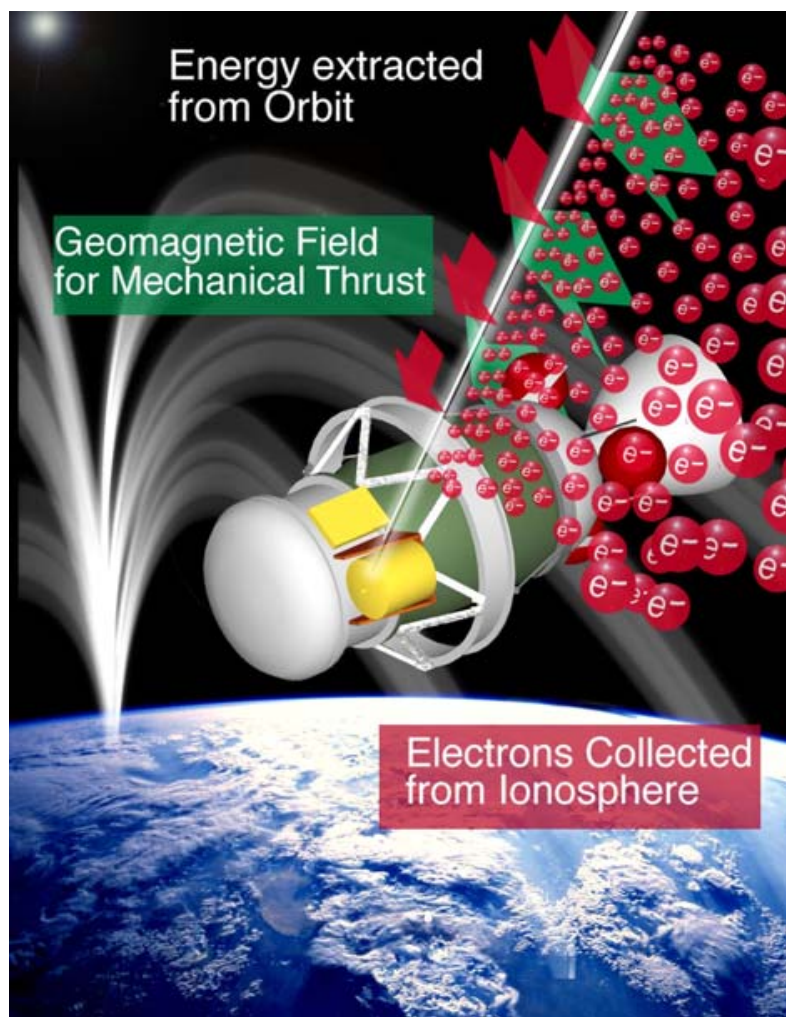

Fig. 1 - Illustration of the electromagnetic concepts used by ProSEDS

larger compared to the original starting altitude. In addition, the thrust will be reduced due to somewhat lower plasma density.

To help estimate the expected changes in performance we use a computer model developed at the University of Michigan called TEMPEST-Tethered Mission Planning and Evaluation Software Tool, which has heritage with previous in-space ED tether missions (TSS- 1 and TSS- -1 R), although all bare tether models are essentially unvalidated because no bare tether experiment has yet flown in space.

One important factor that has become important with the lower initial altitude is the model used for the ionosphere, which governs plasma density and electron temperature. The model used by most is the International Reference Ionosphere (IRI) ${ }^{7}$ and is the standard for predicting ionospheric variations with solar activity and can be used for estimates of future ED tether performance based on predictions of solar activity. However, the IRI provides an estimate of averaged effects only and thus is highly smoothed spatially as compared to what would be observed along the orbit of a spacecraft. Specifically, the IRI predicts a very gradual fall-off in plasma density below the peak density altitude ( $F$-peak) and in some nighttime situations this is not realistic and a more abrupt density change as a function of altitude occurs below the $F$-peak. This means that if an ED tether system passes below the $F$-peak it is possible for current flow to be more 


\section{0-23 July 2003, Huntsville, AL}

dramatically reduced than predicted by the IRI model. This, in turn, would have more significant coupling effects to tether dynamics. Providing a more realistic data set that can be used to identify possible undesired tether dynamics was the goal of the work reported here.

As an alternative to IRI, we are investigating a physics-based ionosphere/plasmasphere model called the Ionospheric Forecast Model (IFM), which is the foundation of a larger initiative called GAIM (Global Assimilation of Ionospheric Measurements) led by Utah State University. ${ }^{8,9,10}$ GAIM is being sponsored by the Air Force (and NOAA) to take real-time observations in order to "nowcast" and ultimately forecast space weather - up to 24 hours! It is believed that the physicsbased model of the ionosphere/plasmasphere system leads to significant improvements in the 3-dimensional plasma density reconstruction, which is especially important in data-sparse regions. Currently, the model extends in magnetic latitude from equatorial to upper mid-latitudes and extends from $100-\mathrm{km}$ to about $20,000-\mathrm{km}$ altitude, covering the ionospheric $E$ - and $F$-regions as well as the plasmasphere. We do not have the IFM available. So, a simplistic approach of scaling the IRI model used in TEMPEST to more closely agree with IFM has been done. It was found that a factor of 0.65 could be used to approximate the IFM model. This is shown in Fig. 2.

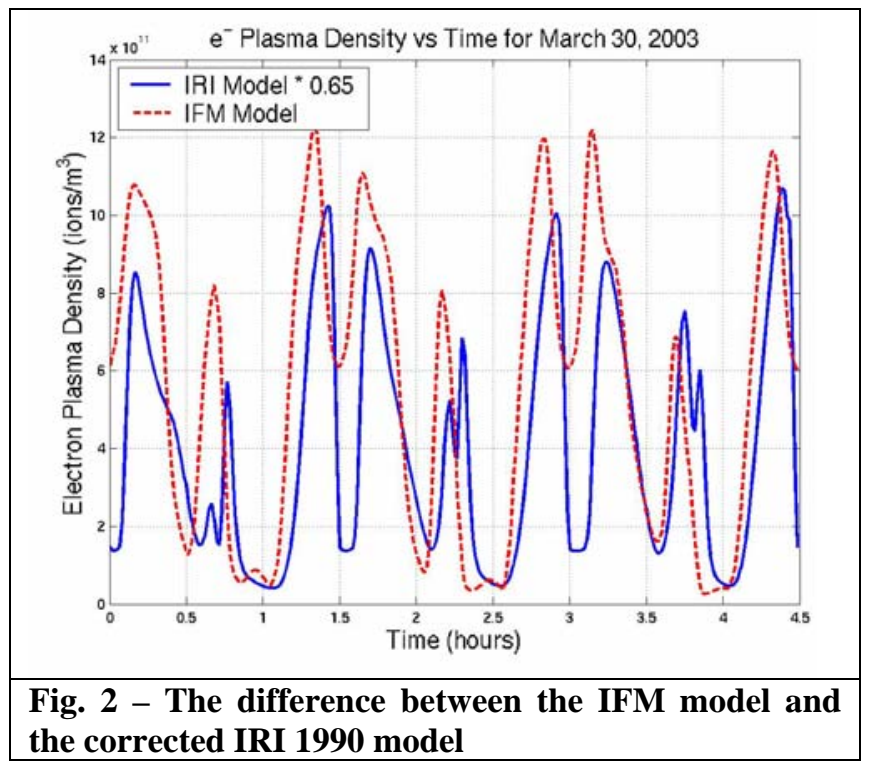

The effects of this new model result in lower currents collected by the bare tether. This, in turn, causes lower tether force and a longer de-orbiting time than without the correction. After taking into consideration the updated ionospheric model and the new mission altitude and tether length, new TEMPEST runs were made.

These TEMPEST simulations were performed for February 3, 2004 (a likely launch date for ProSEDS is winter 2004, so an arbitrary day in February was chosen) with the following specifications: $160 \mathrm{~m}$ of the tether is insulated, $4840 \mathrm{~m}$ of bare aluminum tether in the second tether section, and $7000 \mathrm{~m}$ of non-conducting tether. The plots in Figs. 3 and 4 compare the differences between the previous proposed mission starting altitude $(360 \mathrm{~km})$ with that of the present starting altitude $(285 \mathrm{~km})$. The induced EMF and end tether current can be seen in Figs. $3 a$ and $b$, respectively. The induced EMF at $285 \mathrm{~km}$ is approximately the same as the $360-\mathrm{km}$ case. This can be attributed to the fact that the conducting part of the tether was not shortened, just $2 \mathrm{~km}$ of the non-conducting part was removed. The range of induced EMF is approximately $400-1000 \mathrm{~V}$.

The end tether current was found to be similar at first, but then deviated significantly later on. As the altitude increases, the plasma density increases till it reaches a maximum at $300 \mathrm{~km}$. After that point it begins to decrease back down to near zero again. The plasma densities at the two differing altitudes are approximately the same at $285 \mathrm{~km}$ and $360 \mathrm{~km}$, which is why the end tether currents are similar. The deviation can be attributed to the decrease in altitude because the electron plasma density falls off at lower altitudes. It should be noted that the plasma density does increase until about 60 hours into the mission, which corresponds to about 300 $\mathrm{km}$ as seen in Fig. $3 \mathrm{~b}$.

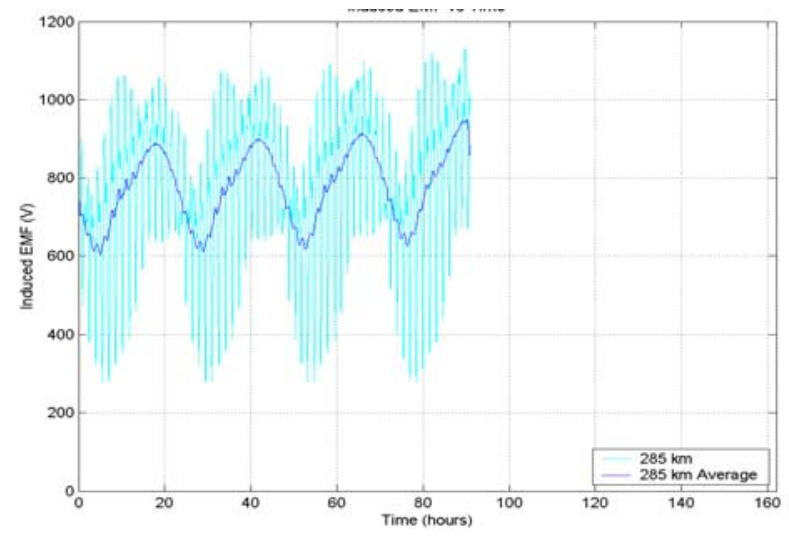

(a)

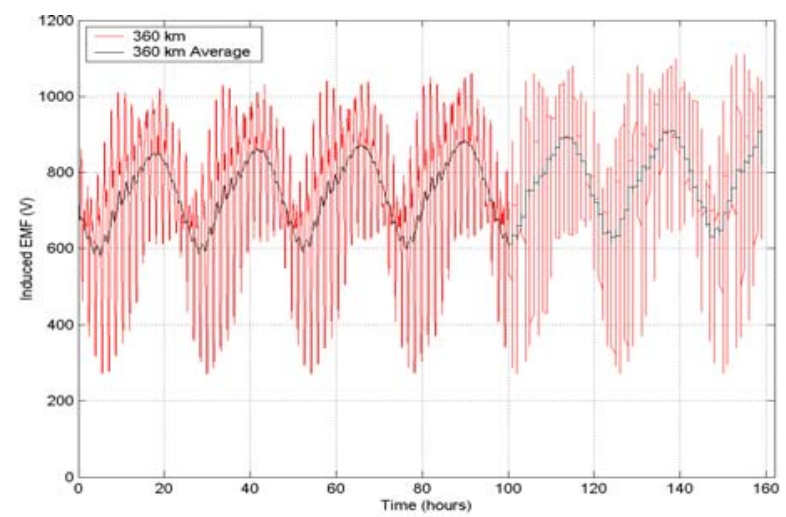

(b) 


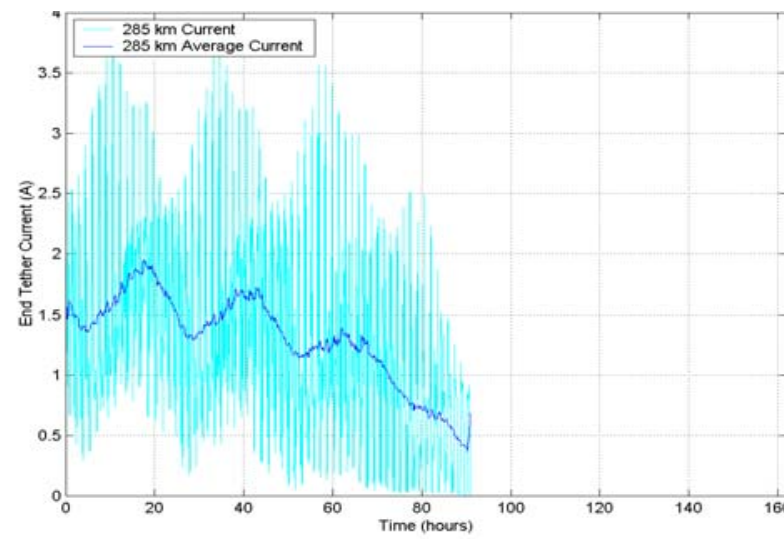

(c)

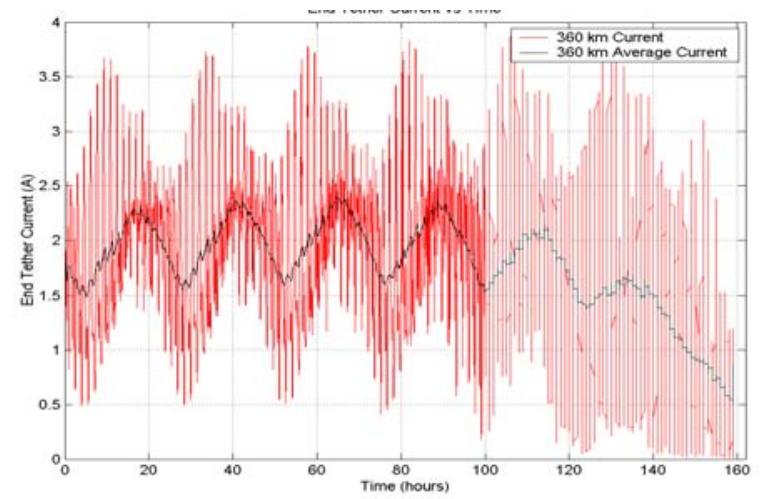

(d)

Fig. 3 - The (a), (b) Induced EMF on the bare teth and the (c), (d) Current at the end of the tether at 28 $\mathrm{km}$ and $360-\mathrm{km}$, respectively

Figure 4a shows that the total time for the mission w de-orbit at $285 \mathrm{~km}$ is 90 hours, while the $360-\mathrm{km}$ run takes 160 hours. This is a significant reduction in the amount of data than can be collected for the mission. Figure $4 \mathrm{~b}$ shows the total amount of drag that is exhibited by the tether and by the atmosphere. It only takes approximately two days for the atmospheric drag to become greater than the tether drag for the $285-\mathrm{km}$ case as opposed to the five and one-half days at $360 \mathrm{~km}$. This means that the amount of time that ProSEDS has to prove that the ED tether technology performs is drastically reduced.

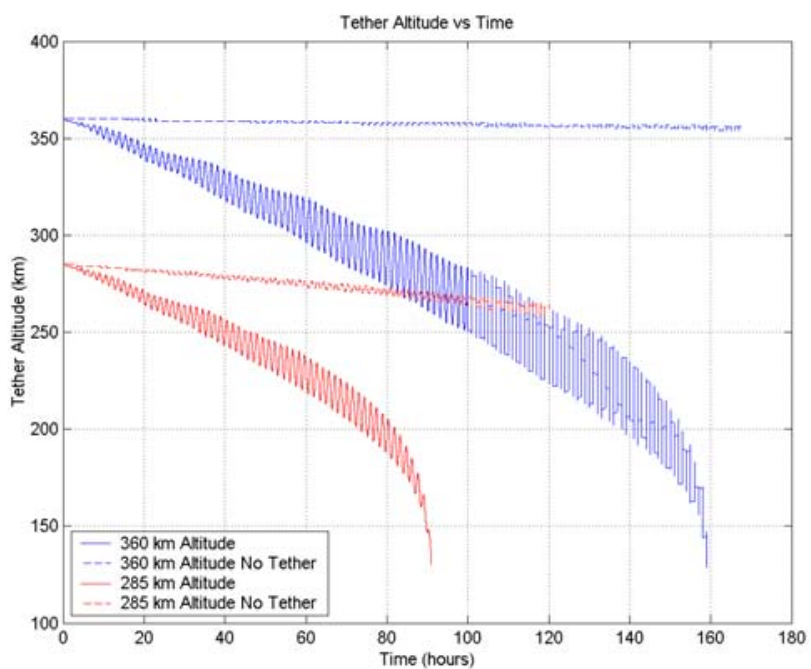

(a)

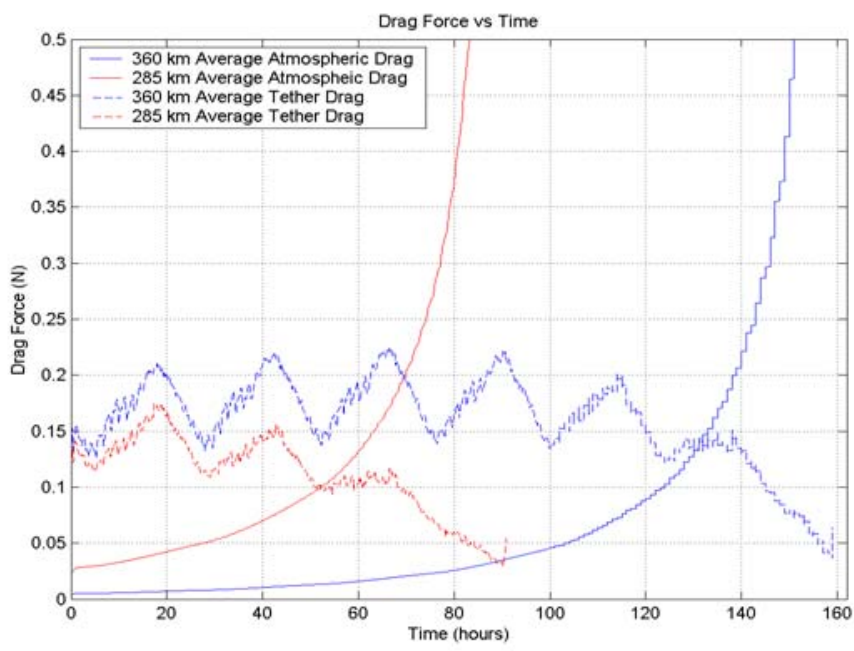

(b)

Fig. 4 - The (a) altitude of the ProSEDS mission and the (b) total drag in the system

\section{Error Analysis}

There are a number of incidences that are not accounted for because of the assumptions that are made with the theory calculations. One major source of error, in particular, occurs during the open-circuit mode of the mission. Since there is a large resistor $(1.5 \mathrm{M} \Omega)$ between the tether and ProSEDS, this mode assumes that nearly zero current flows across the end of the bare tether. This would mean that the current going through ProSEDS must be close to zero total current as well, according to Kirchhoff's current law (KCL). However, this does not prevent current from traveling down the bare tether.

There is still a potential drop due to the induced EMF combined with the ohmic loss along the bare tether. 


\section{0-23 July 2003, Huntsville, AL}

Electron collection near the top of the tether is allowed because the tether potential relative to the plasma is negative. In addition, there is an equal amount of ion thermal current on the bottom part of the tether for the opposite reason. The electron collection occurs along the first few percent of the highest portion of the tether; it then takes the remaining lower portion of the tether for the ion thermal current collection to neutralize the electrons collected in the upper part. As a result of the length it takes the electrons to dissipate and the length of the tether it travels down, an $\mathbf{I} \times \mathbf{B}$ force is produced. TEMPEST simulations shown in Fig. 5a predict an additional potential drop of $10-40 \mathrm{~V}$ due to this "phantom current". Using Ohm's law, the current driven through the tether can be calculated, which ranges from 5-60 mA. The altitude reduction due to this drag force is shown in Fig. 5b, which illustrates how this potential drop and current flow produces approximately a 2-km-per-day altitude reduction of ProSEDS.

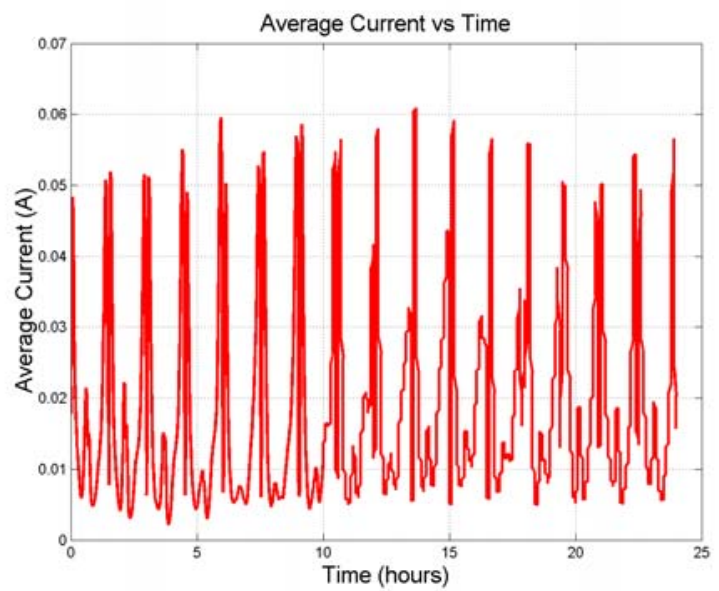

(a)

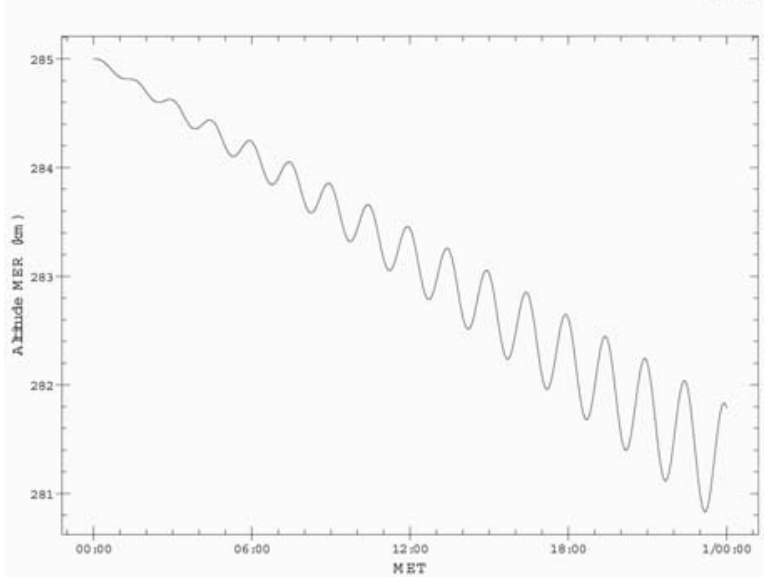

(b)

Fig. 5 - A 24-hour TEMPEST run for ProSEDS in open circuit mode for (a) average current, and (b) altitude drop
Figure 6 shows that there is approximately a 3-4\% error in estimating the total EMF because of this "phantom current".

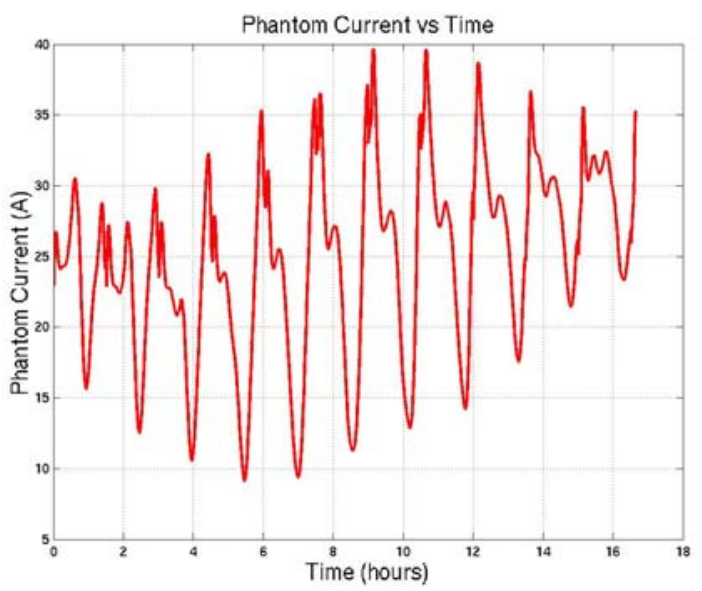

(a)

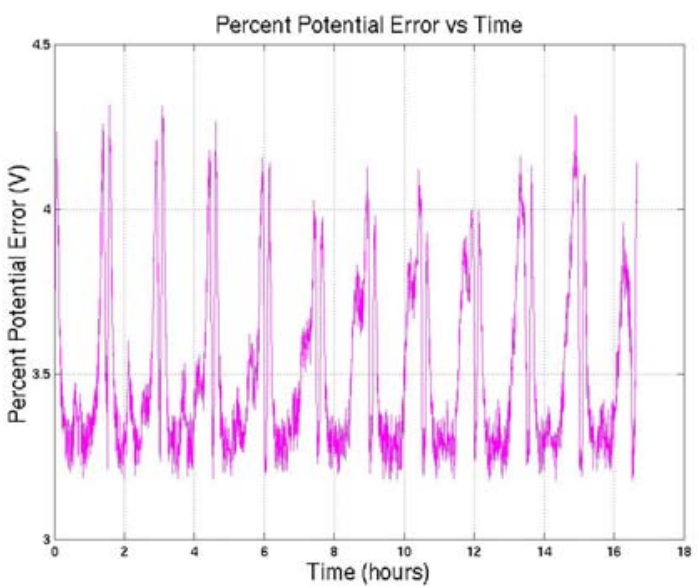

(b)

Fig. 6 - Simulated error of the $\boldsymbol{R}_{\text {upper }}$ section of the circuit in Figure 7. (a) is the total error voltage, and (b) is the percent error relative to the actual EMF.

Other sources of error arise from the fact that there is no perfect model for the ionosphere, which is continually changing, making it nearly impossible to precisely determine the exact plasma density at a given location at any given time. Also, the initial assumption in the TEMPEST runs is that ProSEDS starts with a perfectly circular orbit and there is no tether libration during it orbit.

Another error-producing assumption is the fact that the electron retardation regime for current collection was ignored. There is a small section of tether that is negative in potential (with respect to the plasma) and, hence, still collects electron thermal current. This effect, however, contributes only about $1 \mathrm{~V}$ of error, which is a small percentage of the total tether voltage up to $1060 \mathrm{~V}$. 
20-23 July 2003, Huntsville, AL

A significant effect that is also ignored is the secondary electron emission from the highly negatively biased tether section. This effect occurs when electrons with high energies impact the tether and cause additional electrons to be ejected that can also be collected. This effect can theoretically increase the tether current from 5 $30 \%{ }^{2}$

Another error results from assuming that the electron collection is guided solely by OML theory. There are some space charge effects and magnetic guiding effects that influence the flow of the electrons into the system. These effects would reduce the amount of current that is actually being collected.

One last source of error is from the potential drop on the Delta II nozzle, indicated by the $\phi_{\text {tu }}$ in Fig. 7. It takes a certain amount of potential on the nozzle to collect enough ion current to complete the circuit. This effect is analyzed in the next section.

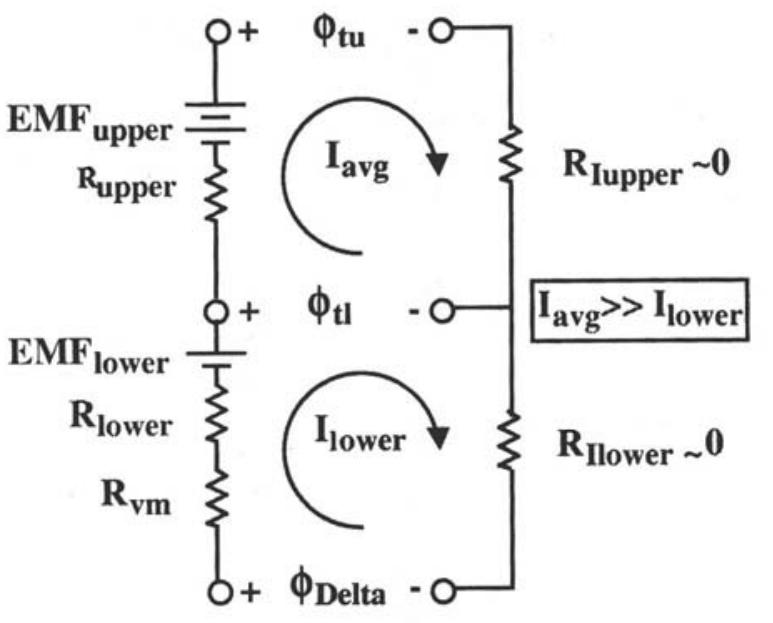

Fig. 7 - Circuit Diagram of a bare tether system

\section{Current Collection}

The analysis of the bare tether system is controlled by a handful of concepts and physical parameters. The tether is made of aluminum, with has a conductivity of $3.5 \times 10^{7}$ $\mathrm{S} / \mathrm{m}$. The resistance of the tether is $\sim 0.05 \Omega / \mathrm{m}$, with an overall length of $5000 \mathrm{~m}$ (160-m insulated, 4840-m bare). At the proposed insertion location in the ionosphere the electron temperature is around $0.1 \mathrm{eV}$ and the density range is between $1 \times 10^{10}$ and $1 \times 10^{12}$ ions $/ \mathrm{m}^{3}$, which makes the Debye length approximately $9.2 \mathrm{~mm}$. Since the Debye length is much larger than the radius of the tether $(1.2 \mathrm{~mm})$ it is assumed that current collection on the tether is in the OML regime. The current collection in the OML regime for a cylinder is given by

$$
\frac{d I_{O M L}}{d y}=e n_{\infty} d\left(\frac{2 e \Delta V}{m_{e}}\right)^{\frac{1}{2}}
$$

where, the $d$ is the diameter of the collecting tether, $e$ is the electron change, $n_{\infty}$ is the ion density, $m_{e}$ is the mass of an electron, and $\Delta V$ is the potential difference between the tether and the plasma. Figure 7 shows a circuit diagram for the entire ProSEDS system. The top half of the diagram represents the bare tether section. $\phi_{\text {tu }}$ is the sheath potential from the top of the tether to the plasma (labeled $\Delta V_{A}$ for anode potential in Fig. 8). $\phi_{\mathrm{tl}}$ is the potential difference from the bottom of the tether to plasma (labeled $\Delta V_{c}$ for cathode potential in Fig. 8). This value is determined by the HCPC (Hollow Cathode Plasma Contactor), which emits a given amount of electrons at the lower end of the tether. Since the current is continuously changing, it is easier to represent the resistive potential drop by taking the entire resistance of the tether multiplied by the average current across the whole tether. Then, using KVL and KCL and Fig. 7, we obtain

$$
\begin{gathered}
\Delta V_{A}+\Delta V_{C}+R_{t} i_{\text {ave }}+R_{v m} i_{\text {end }}=E M F \\
I_{A B}=I_{B C}+I_{C}
\end{gathered}
$$
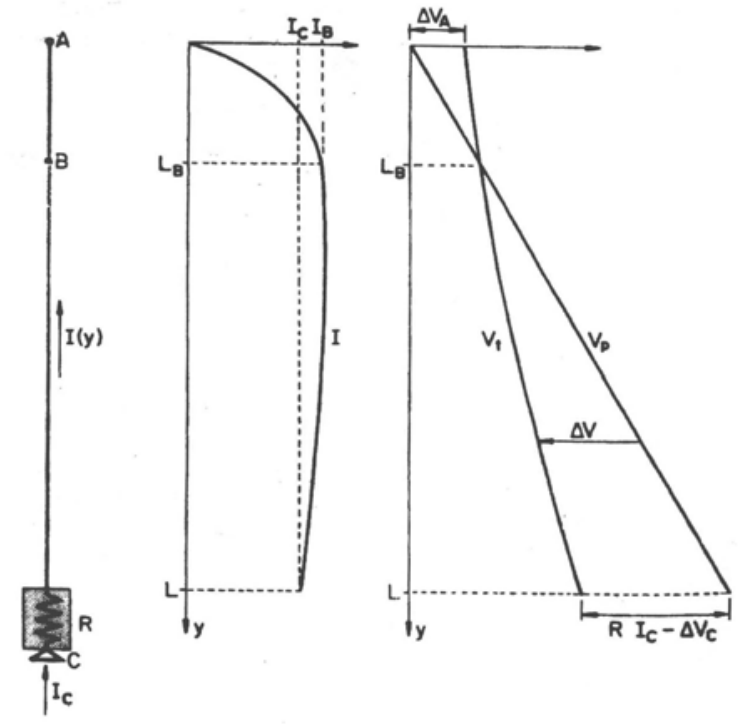

Fig. 8 - Current and Voltage plots over the distance of the tether [From Ref. 3]

After evaluating Eqs. (4) and (5) in the dynamic system, ${ }^{1}$ the results will yield a current and potential profile as seen in Fig. 8. This diagram shows that from the start of the tether till the point $L_{B}$ there is a positive potential bias, and this increases the current through 


\section{0-23 July 2003, Huntsville, AL}

thermal electron collection. Below that point the tether potential becomes negative and the ion thermal current collection takes over. Since it takes a much greater potential difference to collect an equivalent amount of ion current, the total current in the tether is reduced by only a small amount.

The nozzle current collection must also be taken into consideration when calculating the total neutralizing current. When the HCPC is on and the ProSEDS system is short circuited to the tether, the effective ion current collection is only a small fraction of the electrons that are being emitted. However when the tether is in "open circuit" mode and the HCPC is off, ions are the primary contributor to electron emission. During this mode, the tether is connected to the Delta II rocket through a 1.5$\mathrm{M} \Omega$ resistor. Fig. 3a shows that in this mode the tether has up to $1060 \mathrm{~V}$ induced EMF. Using Ohm's law, this effectively reduces the current between the bare tether and the Delta II to $0.733 \mathrm{~mA}$. This amount of electron current must be emitted through the nozzle of the Delta II.

The nozzle is very negatively charged $(\sim 1060 \mathrm{~V})$, and as a result collects no electron thermal current. The only processes that control the electron neutralization when the HCPC is off is ion thermal current collection, ram current collection, and photoemission. The ion thermal current collection is regulated by the size of the sheath around the nozzle. The size of the sheath is determined by the potential from the nozzle to the plasma. The ProSEDS mission will be traveling at mesosonic speeds and therefore will collect ramming ions from the plasma. This ram current collection is determined by the total crosssectional surface area exposed to the oncoming ions, as well as some portion of the effective surface area created by the sheath. The exact portion is not known, so Fig. 9 displays cases where $50 \%$ and $100 \%$ of the sheath was assumed to collect ram current. Photoemission is a smaller effect that occurs when the nozzle is exposed to the sun. The photons collide with the engine bell surface and an electron is emitted. The direction of the flow has a large impact on the electron neutralization because anything in the wake of the plasma will have a significantly reduced plasma density. The ions will be blocked, and the lack of ions will result in the depletion of electrons because they are not being attracted to that region.

The calculation of electron neutralization can be displayed from the following equations

$$
\begin{gathered}
t=\frac{2}{3}\left[\sqrt{\frac{2 e}{m_{e}}} \cdot\left(\frac{\varepsilon_{o} V^{\frac{3}{2}}}{J_{t h} e}\right)\right]^{\frac{1}{2}} \\
\Gamma_{t h}=\frac{1}{4} n_{\infty} \sqrt{\frac{8 T_{e} e}{\pi m_{e}}}
\end{gathered}
$$

For

$$
J_{B o h m}=1.53 \Gamma_{t h}
$$

$$
\frac{t}{r_{O}} \leq 19: \frac{r_{S}}{r_{O}}=\left[\frac{1}{2}+\sqrt{\frac{1}{4}+\frac{t}{r_{O}}}+0.052\left(\frac{t}{r_{O}}\right) H\left(\frac{t}{r_{O}}-0.2\right)\right]^{\frac{1}{2}}
$$

where $H(x) ; H=0$ if $x<0$, else $H=1$. Here, $t$ is the sheath thickness according to the Child-Langmuir law. $J_{t h}$ is the thermal current density, $\Gamma_{t h}$ is the electron thermal flux, $J_{\text {Bohm }}$ is the thermal current density under the Bohm approximation, $r_{0}$ is the radius of the Delta II nozzle, and $r_{\mathrm{s}}$ is the radius of the sheath.

Environmental variables greatly influence the amount of collected current, which includes density, time of day, altitude, electron and ion temperature, and atomic composition.

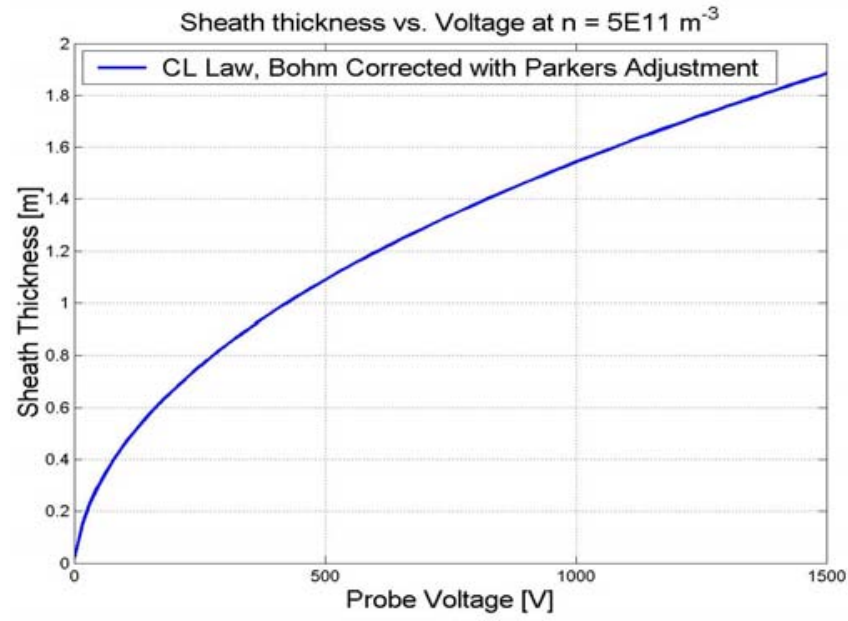

(a)

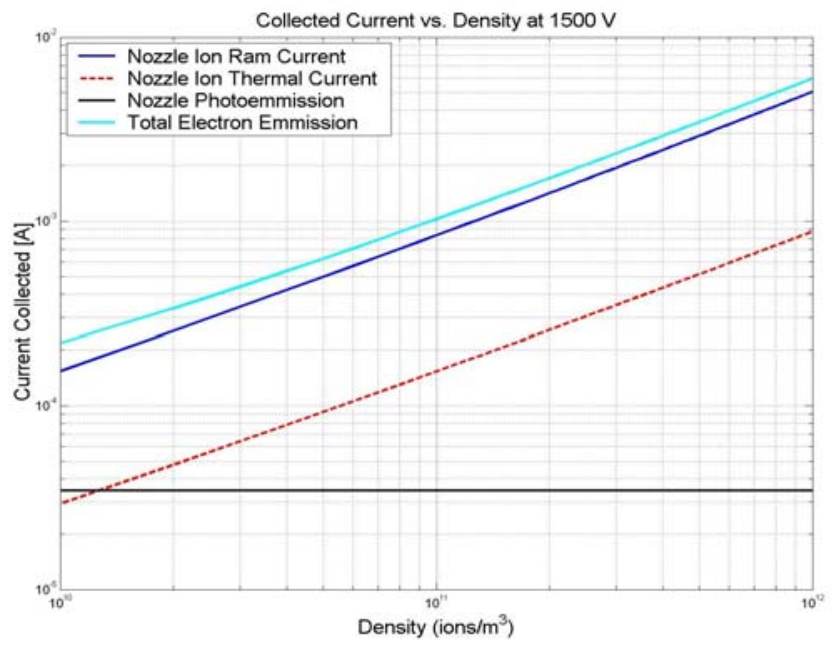

(b) 


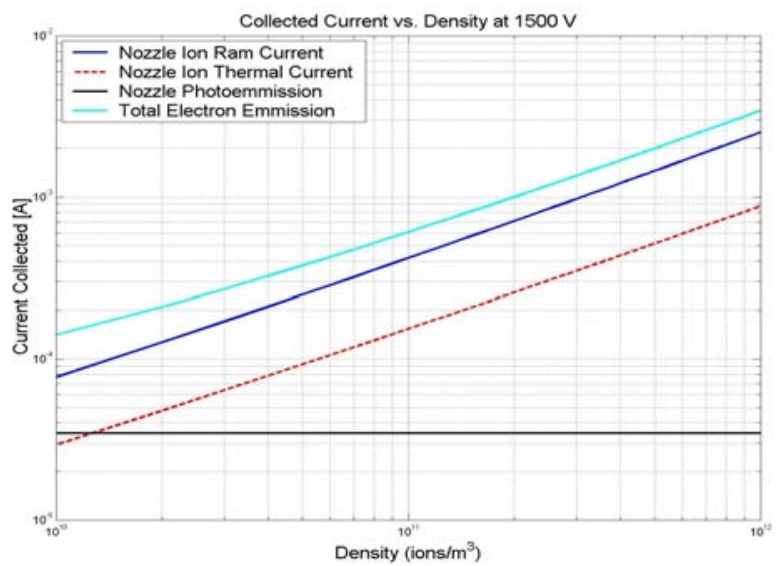

(c)

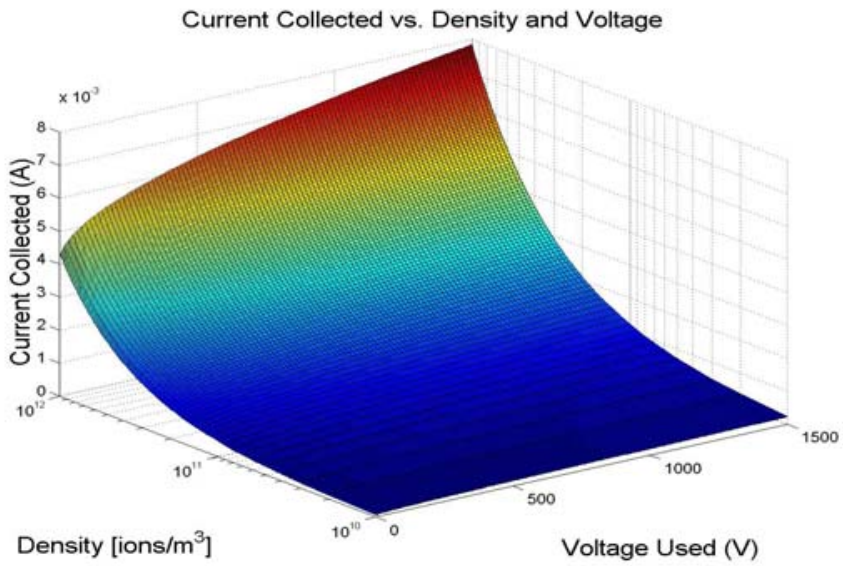

(d)

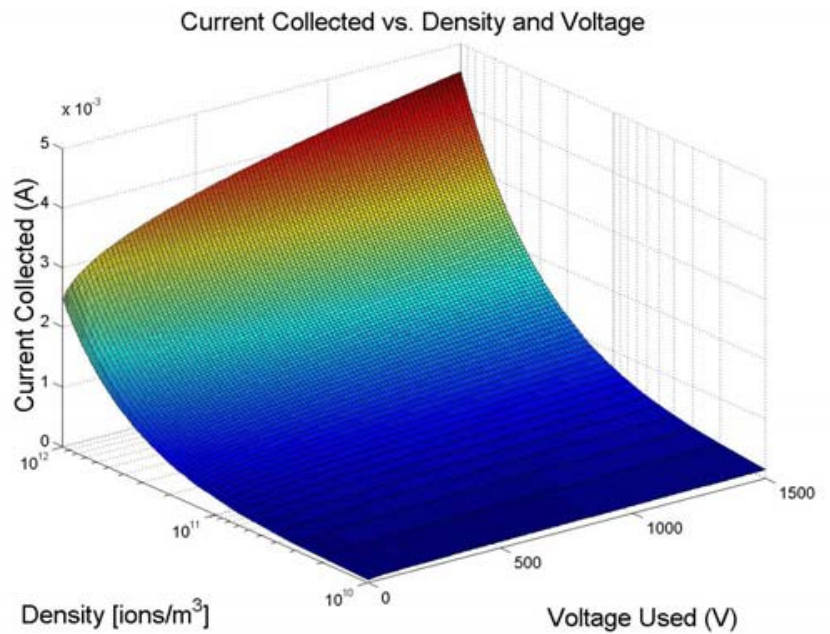

(e)

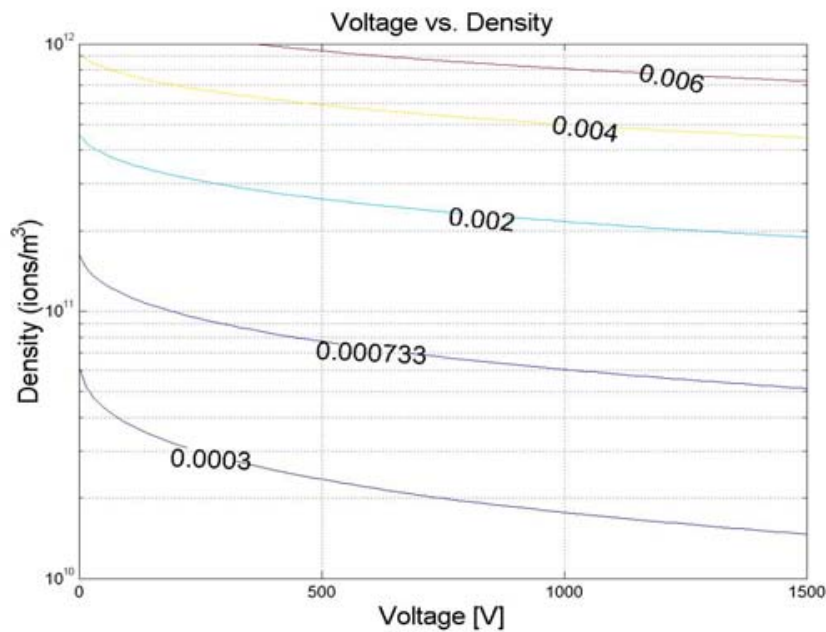

(f)

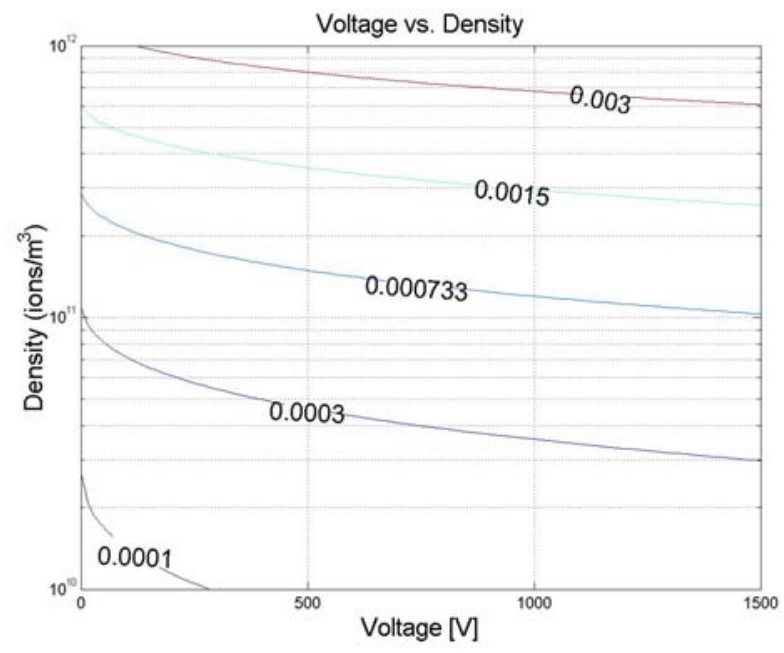

(g)

Fig. 9 - Ion Current collection (electron neutralization) on the nozzle of the Delta II: (a) the sheath, (b), (c) the sum of the components (d), (e) the entire range of collection, and (f), (g) contours of (d), and (e). [(b), (d), and (f) assume $50 \%$ and (c), (e), and (g) assume $100 \%$ of the sheath used to collect ram current.]

It is assumed that the nozzle is in the space charge limited regime since, as shown in Fig. 7a, the sheath size $(1.8 \mathrm{~m})$ is close to the radius of the nozzle $(0.8 \mathrm{~m})$. Ideally, however, the sheath size should be much less than the radius of the nozzle to be in the space charge limited regime. The angular momentum of the particle will play some role on the collection because not all the ions entering the sheath will be collected by the nozzle. The overall effect would be that the space charge predicted collection would just be slightly overestimated. For the calculation of the sheath size $\left(r_{0}\right)$, the Child-Langmuir 
20-23 July 2003, Huntsville, AL

law, Eq. (6), was used. This, combined with the Bohm approximation, Eq. (8), and Parker's approximation, ${ }^{5}$ Eq. (9), yielded the results shown in Fig. 7a. That calculated sheath size, in turn, is used to calculate the amount of ion thermal current collected. The Bohm sheath criterion applies when the object collecting current is in the space charge limited regime and is charged very negative. ${ }^{4}$ All the electron neutralization currents, along with their sums are given in Fig. $7 \mathrm{~b}$ and $7 \mathrm{c}$. The total ion current from Fig. $7 \mathrm{~b}$ and $7 \mathrm{c}$ was used to calculate the collected current across all density and voltage ranges, which resulted in Fig. $7 \mathrm{~d}$ and $7 \mathrm{e}$. Finally, contours at various currents of Fig. $7 \mathrm{~d}$ and $7 \mathrm{e}$ can be seen in Fig. $7 \mathrm{f}$ and $7 \mathrm{~g}$.

The $0.733 \mathrm{~mA}$ of current now can be accounted for given a certain density and voltage from the HCPC.

\begin{tabular}{|c|c|c|c|c|c|}
\hline$n_{e}\left[\right.$ ions $\left./ \mathbf{m}^{3}\right]$ & $\phi_{\mathrm{tu}}[\mathrm{V}]$ & $R t \times I_{\text {ave }}[\mathrm{V}]$ & $\phi_{\text {Delta }}[\mathrm{V}]$ & Total Error $[\mathrm{V}]$ & $\boldsymbol{R}_{\mathrm{vm}} \times I_{\text {end }}[\mathrm{V}]$ \\
\hline $1 \mathrm{E} 10$ & 37 & $200 \times 5.6 \mathrm{E}-4 \approx 0$ & 740 & 777 & 284 \\
\hline $1 \mathrm{E} 11$ & 34 & $200 \times 4.4 \mathrm{E}-3=1$ & 150 & 185 & 877 \\
\hline $1 \mathrm{E} 12$ & 32 & $200 \times 3.8 \mathrm{E}-2=8$ & $\mathbf{0}$ & 40 & 1018 \\
\hline
\end{tabular}

Table 1 - Table of voltages of each component of the system

Using Figs. 7 and 10, the values for each segment of the circuit are now known, and can be checked with KVL to and are given in Table $1 . \phi_{\mathrm{tu}}$ is the tether sheath potential at the upper end of the tether, $R_{t} \times I_{\text {ave }}$ represents the total potential drop across the tether, and $\phi_{\text {Detta }}$ is the potential across the Delta II nozzle. At the points where Fig. 10 was taken the induced EMF was $1061 \mathrm{~V}$. The previous values used with Eq. (4) confirms KVL.

\section{Conclusions}

As a consequence of the starting altitude and tether length change for ProSEDS, the predicted performance will not be as strong as previously estimated. Nevertheless, all ProSEDS objectives should still be achieved, thus validating the effectiveness of ED tethers for propulsion. The induced EMF will remain roughly the same, but the de-orbit time will be reduced by $\sim 40 \%$. The maximum total current collected will be reduced by approximately $10 \%$ and depends on the altitude. The amount of time that ProSEDS has before the atmospheric drag becomes greater than the tether propulsive drag is $\sim 2$ days, which is a quarter of the previous time. The total potential error on the system will be approximately $0-8 \mathrm{~V}$ error for the phantom tether current, 32-37 V for the sheath on the upper portion of the tether, and $0-740 \mathrm{~V}$ for the sheath drop at the nozzle on the Delta II. This results in a total error of as little as 4, but as large as $71 \%$, without the Hollow Cathode operating. With the hollow cathode operating, the sheath error term is less than $40 \mathrm{~V}$.

When the ProSEDS mission launches we will be able to ascertain if our estimates of tether voltage and current were accurate. The actual experimental results from the mission will help us refine simulation programs such as TEMPEST.

\section{Acknowledgments}

The authors would like to thank Ken Welzyn at NASA Marshall Space Flight Center for support in obtaining physical parameters for use in the TEMPEST runs.

\section{References}

1 Johnson, L., B. Gilchrist, E. Lorenzini, N. Stone, and K. Wright, "Propulsive Small Expendable Deployer System (ProSEDS) Experiment: Mission Overview \& Status," AIAA2003-5094, AIAA Joint Propulsion Conf., Huntsville, AL, July 20-23, 2003.

${ }^{2}$ Gilchrist, B. Voronka, N. and, Bilen, S., "ProSEDS "Open Circuit": Characteristics: EMF Measurements and Currents," University of Michigan White Paper, Sept. 9, 1997.

${ }^{3}$ Sanmartin, J. R., Martinez-Sanchez, M., and Ahedo, E., "Bare Wire Anoodes for Electrodynamic Tethers," Journal of Propulsion and Power, Vol. 9, No. 3, May-June 1993, pp. 353360.

${ }^{4}$ Welzyn, K., "ProSEDS Mission Performance Assessments for Lower Starting Altitude," NASA MSFC unpublished internal report, March 3, 2003.

${ }^{5}$ Aguero, V., A Study of Electrical Charging on Large LEO Spacecraft Using a Tethered Satellite as a Remote Plasma Reference, Ph.D. Thesis, Department of Electrical Engineering, Stanford University, 1996.

${ }^{6}$ Parker, L. W., "Plasmasheath-Photosheath Theory for Large High-Voltage Space Structures," Space Systems and their Interactions With Earth's Environment, p. 477, AIAA Press.

${ }^{7}$ Bilitza, D., International Reference Ionosphere 2000, Radio Science, Vol. 36, Iss. 2, 2001, pp. 261-275.

${ }^{8}$ Scherliess, L., R. W. Schunk, J. J. Sojka, and D. C. Thompson, "Development of a Physics-Based Reduced State Kalman Filter for the Ionosphere" 2002 Ionospheric Effects Symposium, Alexandria, VA, May 7-9, 2002.

${ }^{9}$ Schunk, R. W., "Global Assimilation of Ionospheric Measurements (GAIM)," 2002 Ionospheric Effects Symposium, Alexandria, VA, May 7-9, 2002.

${ }^{10}$ Schunk, R. W., L. Scherliess, and J. J. Sojka, "Ionospheric Specification and Forecast Modeling," J. Space. Rockets, Vol. 39, No. 2, March-April 2002. 